BULL. AUSTRAL. MATH. SOC.

VOL. $22(1980), 205-210$.

\title{
A NEW DEFINITION OF VARIATIONAL DERIVATIVE
}

\author{
Eugene P. Hamilton
}

\begin{abstract}
It is shown that the functional $\int_{a}^{b} F\left(x, y, y^{\prime}\right) d x$ fails to possess a variational derivative, contrary to what is claimed by Gelfand and Fomin. A modified definition is given with respect to which the functional does possess a variational derivative.
\end{abstract}

Let $V$ be a space of functions on the interval $[a, b]$ and let $J: V \rightarrow R$. The variational derivative of $J$ is defined by Gelfand and Fomin in [1] and is applied to functionals of the form

$$
J[y]=\int_{a}^{b} F\left(x, y, y^{\prime}\right) d x
$$

on the space $V=D^{1}[a, b] \quad\left(D^{n}[a, b]\right.$ equals the space of functions on $[a, b]$ with $n$ continuous derivatives) to provide elegant proofs of the invariance of Euler's equation and of multiplier rules for solving variational problems with isoperimetric constraints. In this note we show that in fact the above functional may fail to possess a variational derivative. We give a modified definition with respect to which $J$ does have a variational derivative. (Gelfand and Fomin's definition of the variational derivative is essentially the same as Volterra's definition of the first derivative of a functional [2]; Volterra also incorrectly asserts that $J$ has a variational derivative.)

First we state Gel fand and Fomin's definition.

Received 27 February 1980. 
DEFINITION 1 [1]. Let $J[y]$ be a functional depending on the function $y(x)$, and suppose we give $y(x)$ an increment $h(x)$ which is different from zero only in the neighborhood of a point $x_{0}$. Dividing the corresponding increment $J[y+h]-J[y]$ of the functional by the area $\Delta \sigma$ lying between the curve $y=h(x)$ and the $x$-axis we obtain the ratio

$$
\frac{J[y+h]-J[y]}{\Delta \sigma}
$$

Next, we let the area $\Delta \sigma$ go to zero in such a way that both $\max |h(x)|$ and the length of the interval in which $h(x)$ is nonvanishing go to zero. Then, if the ratio (2) converges to a limit as $\Delta \sigma \rightarrow 0$, this limit is called the variational derivative of the functional $J[y]$ at the point $x_{0}$ for the curve $y=y(x)$, and is denoted by $\left.\frac{\delta J}{\delta y}\right|_{x=x_{0}}$.

Gel fand and Fomin claim that the functional (1) has the variational derivative $\frac{\delta J}{\delta y}=F_{y}-\frac{d}{d x}\left(F_{y},\right)$ provided that $F$ has 2 continuous partial derivatives with respect to all of its arguments. A counterexample is the functional $\int_{-1}^{1}\left(y^{\prime}(x)\right)^{2} d x$. We now show that the limit of the ratio (2) does not exist at $x_{0}=0$ for the curve $y \equiv 0$.

Proof. Let $k$ be an infinitely differentiable nonnegative function on $R$ that vanishes off the interval $(-I, I)$ and suppose $k(0)$ is nonzero. Let $k_{c}(x)=c^{p} k(x / c)$ for $0<c<1$ and $p>0$, let $h_{c}(x)$ denote the restriction of $k_{c}$ to $[-1,1]$, and let $\Delta \sigma{ }_{c}$ denote the area under $h_{c}$. Then

$$
\begin{array}{r}
\left.\left.\frac{J\left[h_{c}\right]-J[0]}{\Delta \sigma}=\iint_{-1}^{1}\left(h_{c}^{\prime}\right)^{2} d x\right) /\left(\int_{-1}^{1} h_{c} d x\right)=\left(\int_{-\infty}^{\infty}\left(k_{c}^{\prime}\right)^{2} d x\right) / \iint_{-\infty}^{\infty} k_{c} d x\right) \\
=c^{p-2}\left(\left[\int_{-\infty}^{\infty}\left(k^{\prime}(u)\right)^{2} d u\right) /\left(\int_{-\infty}^{\infty} k(u) d u\right)\right) .
\end{array}
$$

The above quotient is positive since $k$ is nonnegative and $k(0)$ is nonzero. As $c$ approaches zero, $\Delta \sigma_{c}$ approaches zero as in Definition 1. For $0<p<2$, the quotient approaches infinity as $c$ approaches zero; 
hence $J$ does not have a variational derivative at $x=0$ for the curve $y \equiv 0$.

We now give a modified definition with respect to which $J$ does have a variational derivative.

DEFINITION 2. Let $J[y]$ be a functional depending on the function $y(x)$, and suppose we give $y(x)$ a $C^{\infty}$ increment $h(x)$ with the following properties:

(1) $h$ does not change sign;

(2) $h\left(x_{0}\right) \neq 0$;

(3) $h=0$ outside a neighborhood of $x_{0}$.

Dividing the corresponding increment $J[y+h]-J[y]$ of the functional by the area $\Delta \sigma$ lying between the curve $y=h(x)$ and the $x$-axis, we obtain the ratio

$$
\frac{J[y+h]-J[y]}{\Delta \sigma}
$$

Next, we let the area $\Delta \sigma$ go to zero in such a way that $\max h^{(p)}(x) \rightarrow 0$ for each nonnegative integer $p$ and the length of the interval in which $h(x)$ is nonvanishing go to zero. Then, if the ratio (2) converges to a limit as $\Delta \sigma \rightarrow 0$, this limit is called the variational derivative of the functional $J[y]$ at the point $x_{0}$ for the curve $y=y(x)$, and is denoted by $\left.\frac{\delta_{J}}{\delta y}\right|_{x=x_{0}}$. Next, we state

THEOREM. Let $F: R^{3} \rightarrow R$ have bounded third partial derivatives with respect to each of its arguments and let $J[y]=\int_{a}^{b} F\left(x, y(x), y^{\prime}(x)\right) d x$. Then $J$ has a variational derivative given by $F_{y}-\frac{d}{d x}\left(F_{y},\right)$ for each $y \in D^{3}[a, b]$ and $x_{0} \in(a, b)$.

Proof. Let $h$ be a smooth function on $[a, b]$ supported in the open subinterval $I$ containing $x_{0}$. Then 


$$
J[y+h]-J[y]=\int_{a}^{b}\left\{F\left(x, y+h, y^{\prime}+h^{\prime}\right)-F\left(x, y, y^{\prime}\right)\right\} d x
$$

(3) $J[y+h]-J[y]=\int_{a}^{b}\left(F, h+F, h^{\prime}\right) d x$

$$
+\frac{1}{2} \int_{a}^{b}\left(\bar{F}_{y y} h^{2}+2 \bar{F}_{y y^{\prime}} h h^{\prime}+\bar{F}_{y^{\prime} y^{\prime}}\left(h^{\prime}\right)^{2}\right) d x,
$$

where the overbar indicates that the corresponding derivatives are evaluated along certain intermediate curves, that is,

$$
\dot{\bar{F}}_{y y}=F_{y y}\left(x, y+\theta h, y^{\prime}+\theta h^{\prime}\right), 0<\theta<1 \text {, }
$$

and similarly for $\bar{F}_{y y^{\prime}}$ and $\bar{F}_{y^{\prime} y^{\prime}}$. (To see this note that $J$ is twice Fréchet differentiable,

$$
\delta^{2} J[y, h]=\int F_{y y^{\prime}} h^{2}+2 F_{y y^{\prime}} h h^{\prime}+F_{y^{\prime} y^{\prime}}
$$

and by Taylor's Theorem with remainder applied to $J$,

$$
J[y+h]-J[y]=\delta_{J}[y, h]+\delta^{2} J[y+\theta h, h]
$$

for some constant $\theta$ between 0 and 1 .) By integration by parts we have, using the fact that $h(a)=h(b)=0$,

$$
\begin{aligned}
\int_{a}^{b} F_{y^{\prime}} h^{\prime} d x & =-\int_{a}^{b} \frac{d}{d x}\left(F_{y^{\prime}}\right) h d x \\
\int_{a}^{b} 2 \bar{F}_{y y^{\prime}}, h h^{\prime} d x & =\int_{a}^{b} \bar{F}_{y y^{\prime}} \frac{d}{d x}\left(h^{2}\right) d x=-\int_{a}^{b} \frac{d}{d x}\left(\bar{F}_{y y^{\prime}}\right) h^{2} d x, \\
\int_{a}^{b} \bar{F}_{y^{\prime} y^{\prime}}\left(h^{\prime}\right)^{2} & =-\int_{a}^{b}\left[\frac{d}{d x}\left(\bar{F}_{y^{\prime} y^{\prime}}\right) h^{\prime}+\bar{F}_{y^{\prime} y^{\prime}} h^{\prime \prime}\right] h .
\end{aligned}
$$

Substituting these relations into (3) we obtain

$$
J[y+h]-J[y]=I_{1}+I_{2}+I_{3}
$$

where 


$$
\begin{aligned}
& I_{1}=\int_{I}\left(F_{y}-\frac{d}{d x} F_{y^{\prime}}\right) h d x, \\
& I_{2}=\int_{I}\left(\bar{F}_{y y}-\frac{d}{d x} \bar{F}_{y y^{\prime}}\right) h^{2} d x, \\
& I_{3}=-\int_{I}\left[\frac{d}{d x}\left(\bar{F}_{y^{\prime} y^{\prime}}\right) h^{\prime}+\bar{F}_{y^{\prime} y^{\prime}} h^{\prime \prime}\right] h d x .
\end{aligned}
$$

By our assumptions on $F,\left(I_{2}+I_{3}\right) / \Delta \sigma \rightarrow 0$ as

$$
\max \left(|h(x)|,\left|h^{\prime}(x)\right|,\left|h^{\prime \prime}(x)\right|\right) \rightarrow 0 \text {. }
$$

By the mean value theorem for integrals,

$$
I_{1}=\left(F_{y}-\frac{d}{d x} F_{y^{\prime}}\right)(c) \int_{I} h d x
$$

for some $c \in I$. As $\Delta \sigma=\int h d x$, and $h \rightarrow 0$, in such a way that $\max \left(|h(x)|,\left|h^{\prime}(x)\right|,\left|h^{\prime \prime}(x)\right|\right) \rightarrow 0$ and the support of $h$ shrinks to $x_{0}$, $c \rightarrow x_{0}$, and hence

$$
\frac{J[y+h]-J[y]}{\Delta \sigma} \rightarrow\left(F_{y}-\frac{d}{d x} F_{y^{\prime}}\right)\left(x_{0}, y\left(x_{0}\right), y^{\prime}\left(x_{0}\right)\right)
$$

With this new definition the proofs of the invariance of Euler's equation and multiplier rules in [1] become valid. Moreover it is easy to see that the more general functional

$$
J[y]=\int_{a}^{b} F\left(x, y(x), y^{\prime}(x), \ldots, y^{(n)}(x)\right) d x
$$

has a variational derivative for the curve $y=y(x)$ provided that $y$ has $2 n+1$ continuous derivatives and $F$ has bounded $(n+2)$ nd partial derivatives with respect to each of its arguments.

\section{References}

[1] I.M. Gelfand, S.V. Fomin, Calculus of variations (translated by Richard A. Silverman. Prentice-Hall, Englewood Cliffs, New Jersey, 1963). 
[2] Vito Voterra, Theory of functionals and of integral and integrodifferential equations (Blackie \& Son, London, 1931; reprinted, Dover, New York, 1959).

Department of Mathematics, Washington College, Chestertown, Maryland 21620 ,

USA. 